\title{
Still Trying to Understand Methotrexate
}

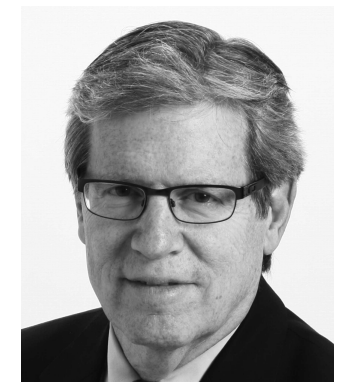

A classic and often quoted mode of progress in medical therapeutics is the connection from bench to bedside. In fact, many of our potent new therapeutic agents such as biologic drugs and Janus kinase inhibitors have been made possible because of biologic and mechanistic insights leading to these new treatments for complex autoimmune diseases.

But what about research that evolves in the opposite direction? That is, first comes the observation of a particular clinical response phenomenon and then researchers circle back and try to determine what is driving the phenotypic patterns that are observed in the clinic. This is actually what has occurred with research into the possible mechanisms of action of methotrexate (MTX).

This amazing drug has been in use for more than 60 years. It was a designer agent for the purpose of competing with intracellular reduced folates, ubiquitous cofactors for a host of critical enzymatic activities. The rationale was that if MTX could fool the cell into uptake, because of its resemblance to the necessary folate substrate, this Trojan horse could then interfere with normal function and serve as a useful tool for oncologists to use against various cancers.

But before too long the drug was used for rapidly proliferating cutaneous cells (psoriasis) and then anecdotal, and later focused, studies to treat various forms of arthritis in the 1970s and 1980s. Clinicians were desperate for effective drugs that worked for challenging conditions such as psoriasis, as well as psoriatic and rheumatoid arthritis (RA).

Thus, MTX had been used for years before its precise mechanism of action was understood, or how that mechanism could work to treat a wide variety of diseases. This historical sequence is entirely understandable. A fellow with his hair on fire doesn't ask about the chemical bonding properties of the water needed to put out the blaze.

As already implied, much of what we know about MTX was inherited from researchers who studied the drug for applications to treat cancer. Chabner and colleagues were the first to describe MTX as a prodrug that needed intracellular conversion to its polyglutamated form in order to be retained within the cell for a therapeutic outcome ${ }^{1}$. At about the time that Chabner described the polyglutamation of MTX, the drug began to be widely used in the United States in the treatment of RA.

There is now some evidence that MTX-polyglutamate (MTX-PG) levels in patients with RA may be related to efficacy ${ }^{2,3,4,5,6,7}$, although not all investigators have reached the same conclusions ${ }^{8}$. Nevertheless, researchers are rightfully examining the factors that affect the accumulation of MTX-PG, which could lead to important insights into their contribution to the therapeutic effects of MTX. Because MTX survives in the circulation for less than $24 \mathrm{~h}$ in subjects with normal renal function, it is relevant and potentially useful to examine the intracellular levels of MTX-PG and the factors that might affect their accumulation.

Any nucleated cell will need to take up folic acid, and therefore be a candidate to take up MTX. The only reliably and easily accessible cell type in which measurements of MTX-PG can occur is the circulating red blood cell (RBC). Because the circulating RBC no longer have a nucleus and no longer take up MTX, it is apparent that any intracellular level of PG must reflect their status prior to their release into the circulation. That is, MTX-PG in the RBC have accumulated while that cell resided in the bone marrow in a nucleated precursor form. Because circulating RBC are assumed to have a half-life of about 120 days, the measurement of RBC MTX-PG levels at any given time will be an amalgam of recently released and older cells, reflecting cellular exposures that have occurred over a period of months. (Think of light from a star seemingly experienced in real-time but actually reflecting a past reality.)

These temporal issues are worth considering when we try to correlate RBC MTX-PG levels with any clinical or demographic variables that are continuous in real time. That is, clinical response and MTX dosage should ideally be at a steady state over a period of at least 4 months so that all the circulating RBC have conceptually been exposed to a stable dose of the drug.

See MTX-PG levels in RA, page 2167

Personal non-commercial use only. The Journal of Rheumatology Copyright (c) 2014. All rights reserved. 
With the above in mind, den Boer and colleagues report in this issue of The Journal on the association of RBC MTX-PG levels with demographic, clinical, and biochemical factors associated with RBC MTX accumulation ${ }^{9}$. In addition, they report on a variety of single nucleotide polymorphisms of intracellular folate pathway enzymes that have the potential to be affected by intracellular MTX-PG. They used a derivation cohort of 97 patients and a validation cohort of 247 patients for their analysis. The cohorts differed somewhat in creatinine clearance, disease activity, and simultaneous use of other disease-modifying antirheumatic drugs. The authors examined independent MTX-PG subspecies (polyglutamates 1 through 5 added to MTX) for each clinical/demographic variable measured in the derivation and validation groups.

MTX polyglutamation occurs sequentially with the addition of longer chain glutamic acid moieties over time, with the predominant species being MTX-PG3. This process takes up to 28 weeks to reach steady state as described by Dalrymple, et $a l^{10}$ and is dependent on cellular uptake, as well as the enzyme folyl polyglutamate synthetase (Figure 1). The authors report that only $21 \%$ of the variability in the MTX-PG in the derivation cohort and "up to $10 \%$ " of the variability in the larger validation cohort could be explained by the factors they had measured. The mean variability in MTX-PG that could be predicted by the combined cohorts was $16 \%$.

Considering this relatively small contribution of RBC
MTX-PG levels to the model variables used to predict intracellular MTX-PG, it may be useful to consider the principles of accumulation of MTX-PG. Den Boer and colleagues measured RBC MTX-PG at 3 months after MTX initiation, which is before nucleated precursors of all the RBC in the sample would have been exposed to MTX in the bone marrow ${ }^{9}$. Although the doses of MTX administered in the 2 cohorts were different, the drug was mostly administered orally. Given the well-described diminished bioavailability of MTX when doses of $>15 \mathrm{mg}$ weekly are administered by mouth, the actual exposure of the subjects in the larger validation cohort, which received a starting and maintenance dose of $25 \mathrm{mg}$, is unclear. In addition, perhaps too much is made of slight differences the authors observed in the various MTX-PG subspecies, given the other vagaries described, including a fixed oral dose in the validation cohort versus a dose determined by the treating physician in the derivation cohort.

That is not to say that the study is without merit. The authors deserve considerable credit for their attempts to better understand the key intracellular events associated with the activity of our most commonly used and effective medication.

An ideal investigation of clinical, demographic, and other influences on the accumulation of MTX-PG would examine a range of doses of the medication administered subcutaneously and at clinical steady state over a period of administration of at least 4 months. Until this hypothetical
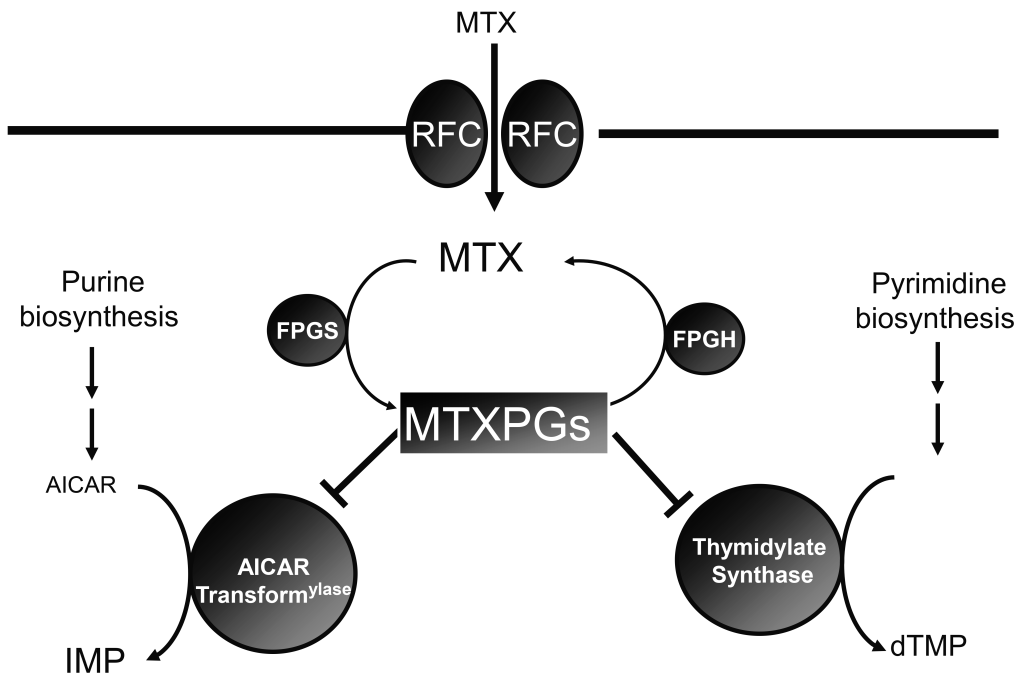

Figure 1. Methotrexate (MTX) enters the cell by means of an active transport mechanism at the reduced folate carrier (RFC). Once MTX has entered the cell, glutamic acid moieties accumulate and are catalyzed by the enzyme folyl polyglutamate synthetase (FPGS). Methotrexate polyglutamates are deglutamyalated by folyl polyglutamate hydrolase (FPGH). It is the intracellular levels of MTX-PG that determine the efficacy of MTX. MTX-PG: methotrexate polyglutamate; AICAR: Aminoimidazole carboxamide ribonucleotide; IMP: inosine monophosphate; dTMP: thymidine-5'-phosphate.

Personal non-commercial use only. The Journal of Rheumatology Copyright @ $\subset$ 2014. All rights reserved. 
trial is conducted, the incremental insights provided by trials, like those of den Boer and colleagues, add to our understanding but raise many other questions as well. Our most reliable and commonly used drug for the treatment of patients with RA and many other rheumatologic conditions has not yet revealed all its secrets.

\author{
JOEL M. KREMER, MD, \\ Pfaff Family Professor of Medicine, \\ Albany Medical College, \\ Director of Research, \\ The Center for Rheumatology, \\ Albany, New York, USA.
}

Address correspondence to Dr. J.M. Kremer, Center for Rheumatology, 1367 Washington Ave., Albany, New York 12206, USA.

E-mail: jkremer@joint-docs.com

\section{REFERENCES}

1. Chabner BA, Allegra CJ, Curt GA, Clendenninn NJ, Baram J, Koizumi S, et al. Polyglutamation of methotrexate. Is methotrexate a prodrug? J Clin Invest 1985:76:907-12.

2. Angelis-Stoforidis P, Vajda FJ, Christophisis N. Methotrexate polyglutamate levels in circulating erythrocytes and polymorphs correlate with clinical efficacy in rheumatoid arthritis. Clin Exp Rheumatol 1999; 17:313-20.

3. Dervieux T, Furst D, Lein DO, Capps R, Smith K, Walsh M, et al. Polyglutamation of methotrexate with common polymorphisms in reduced folate carrier, aminoimidazole carboxamide ribonucleotide transformylase, and thymidylate synthase are associated with methotrexate effects in rheumatoid arthritis. Arthritis Rheum 2004;50:2766-74.
4. Dervieux T, Kremer J, Lein DO, Capps R, Barhan R, Meyer G, et al. Contribution of common polymorphisms in reduced folate carrier and gamma-glutamylhydrolase to methotrexate polyglutamate levels in patients with rheumatoid arthritis. Pharmacogenetics 2004;14:733-9.

5. Dervieux T, Furst D, Lein DO, Capps R, Smith K, Walsh M, et al. Pharmacogenetic and metabolite measurements are associated with clinical status in patients with rheumatoid arthritis treated with methotrexate: results of a multicentred cross sectional observational study. Ann Rheum Dis 2005;64:1180-5.

6. Dervieux T, Greenstein N, Kremer J. Pharmacogenetic and metabolic biomarkers in the folate pathway and their association with methotrexate effects during dosage escalation in rheumatoid arthritis. Arthritis Rheum 2006;54:3095-103.

7. Dervieux T, Zablocki R, Kremer J. Red blood cell methotrexate polyglutamates emerge as a function of dosage intensity and route of administration during pulse methotrexate therapy in rheumatoid arthritis. Rheumatology 2010;49:2337-45.

8. Stamp LK, O'Donnell JL, Chapman PT, Zhang M, Frampton C, James J, et al. Determinants of red blood cell methotrexate polyglutamate concentrations in rheumatoid arthritis patients receiving long-term methotrexate treatment. Arthritis Rheum 2009;60:2248-56.

9. den Boer E, de Rotte MC, Pluijm SM, Heil SG, Hazes JM, de Jonge R. Determinants of erythrocyte methotrexate polyglutamate levels in rheumatoid arthritis. J Rheumatol 2014;41:2167-78.

10. Dalrymple JM, Stamp LK, O'Donnell JL, Chapman PT, Zhang M, Barclay ML. Pharmacokinetics of oral methotrexate in patients with rheumatoid arthritis. Arthritis Rheum 2008;58:3299-308.

J Rheumatol 2014;41:2099-101; doi:10.3899/jrheum.141081 